\title{
Real Options in Sustainable Development Projects
}

\author{
Krzysztof S. Targiel \\ Department of Operations Research \\ University of Economics in Katowice \\ Katowice, Poland
}

\begin{abstract}
Sustainable development involves a change in the status quo. The most effective way for this change is driving projects. In a situation of limited resources, it is essential to choose the right project. Selection is based on an evaluation of the project. Traditionally, the evaluation of development projects is made on the basis of financial criteria. In the context of sustainable development, the social and environmental aspects must also be considered. Contemporary project valuation takes into account not only the static planned cash flows but also opportunities that may appear, known as real options. This paper describes a method for the valuation of development projects, taking into account the above aspects of valuation.
\end{abstract}

\section{Introduction}

Any development binds to a change in the status quo. This also applies to sustainable development. Spotted opportunities, perceived threats cause that we want to change this state. The most effective way for this change is conducting projects. In a situation of limited resources, it is essential to choose the right project. Selection is based on an evaluation of the project. In the context of sustainable development, it should not only be a financial assessment, but also take into account social and environmental factors.

Traditional project evaluation is based on the discounted cash flow method (DCF). The main measure of effectiveness is the Net Present Value (NPV). When this value is positive, the project is approved, when negative, it is rejected, but this approach sometimes leads to the abandonment of profitable projects. The reason for this is that the DCF method does not take into account the role of managerial flexibility. The Project Manager has the right to take action as appropriate. This situation is called a real option. Using Real Option Valuation (ROV), we can provide a quantitative measurement for this situation.

In ROV, methods from the financial market, such as the Black-Scholes model [1] or the Cox-RossRubinstein model (CRR)[2] were used first. An approach based on Monte Carlo simulations [3] has also been used. The CRR model is based on the binomial tree. This approach was also adopted in Guthrie's work [4], on which this study is based.
The standard approach in the valuation of real options is based on one factor, called the state variable. There have been attempts to take into account many state variables. The first attempt, on the basis of financial options, was made by Boyle [5], who took into account two assets. Mun [6] described the commercial solution as having such possibilities. Guthrie [4] also described problems for which it was necessary to take into consideration a number of variables. In these solutions, different criteria were brought to a common financial denominator.

ROV-based approach allows not only on the valuation of the project, but also can assist in the decision making process. For example, if the project is divided into phases, there is the problem of selecting the start of the next stage. The same is in the case with the possibility of delaying the start of the project. Should be chosen the right moment it began. If the result of the project depends on some external factors, that changes in time, it is possible to help in selection with ROV methods. The condition is that these factors can be modeled using stochastic processes.

This paper presents the use of Real Option Valuation (ROV) methods for decision support in sustainable development projects. The nature of the problem is the need to use multiple state variables, which leads to problems considered in the multicriteria analysis. This paper presents solution of such multi-criteria problem with two state variables. The proposed solution considers two aspects, which are especially important in the context of sustainable development namely social aspects and financial aspects. The first section presents the Defer Options that may arise in project management. The next section describes a multi-criteria approach in Real Options valuation, which could be applicable in presented situation. The last section is a numerical example.

\section{Problem formulation}

Project management produces an environment in which many decisions are made. Many project management methodologies, such as PRINCE2 [7], for example, recommend dividing the project into stages. This raises the problem of decision making, 
the choice of the start of the next steps. A similar situation occurs when we are able to delay the start of the project. This is a classic example of the defer options considered by Ingersoll and Ross [8].

If start is planned in advance, the situation is static; the decision maker is not able to react to changes in the environment and in the same project. If extending the duration of the project is allowed and the decision maker is allowed to freely decide about the start times, a completely new situation is raised, as presented in Figure. 1. The decision maker may start the project (decision $\boldsymbol{A}$ ), then move from the current state (initialization of project) to the last state (end of project). The decision maker may wait (decision $\boldsymbol{W}$ ), but then the project will remain in its starting state. After one period, the situation is repeated. The decision maker may start the project (decision $\boldsymbol{A}$ ), then move from the current state (initialization of project) to the last state (end of project). The decision maker may also wait (decision $\boldsymbol{W})$, but in this case the project will fail.

The ability to delay the project for one period time creates a real option, expanding managerial flexibility. Project manager has the possibility to use it freely, depending on the situation in environment. If the current environment is favorable, a project manager will begin the project, if it is not beneficial can wait on the development of the situation.

The increase in flexibility is associated with the problem of when to use his option. In the present case it is the problem of choosing the best moment to start the project. Is it better to start a project now or rather wait for development of the situation.

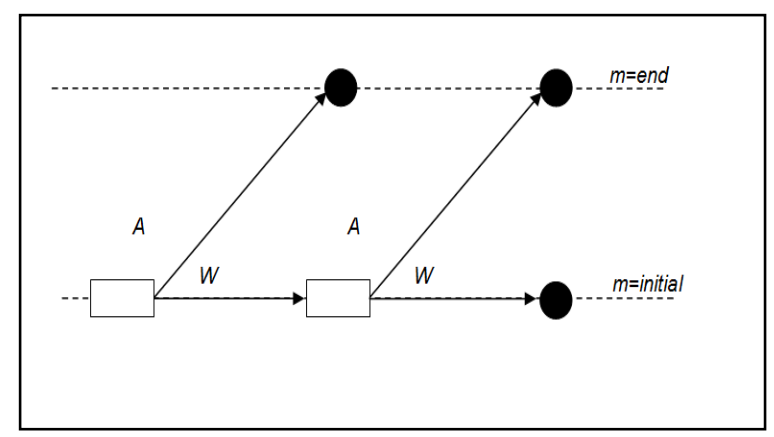

Figure 1. Considered situation

The solution to this problem requires a multicriteria approach. About this approach will be extended methodology ROV. The solution to this problem requires a multi-criteria approach. About this approach will be extended methodology ROV. Those methods are based on backward induction. called dynamic programming. They will be used in the presented method. It may be that historical data do not indicate positive developments, or on the contrary, there is a chance in the future to obtain the benefits of the project started. This requires a quantitative assessment of the situation. These problems are solved by methods ROV, if the factor has only the financial aspect. In projects of sustainable development may be insufficient approach. In this case, should be consider the achievement by the project other non-financial results.

The results of the project, in terms of its value, depend on certain factors, which may be:

- Economic factors (commodity prices, exchange rates)

- Social factors

- Environmental factors

according to the principles of sustainable development. If we consider more than one factor that leads to usability and design considerations in many areas, the problem is converted from a simple valuation to a multi-criteria evaluation problem.

This approach is discussed in the literature. Bednyagin and Gnansounou [9] consider the spillover benefits of the $\mathrm{R} \& \mathrm{D}$ program on thermonuclear fusion technology. The socioeconomic benefits are higher than the expected costs. The framework for project-level decisions, leading to more sustainable management and development, is proposed in [10]. The ecological, economic and social sources of landscape valuation are discussed in [11]. Heidkamp's paper [12] proposes a theoretical framework for the integration of economic aspects and environmental aspects into the decision making process for sustainable development strategies. The paper [13] incorporates sustainable development criteria into decision making processes within the industry. Renewable energy investment projects as an integral part of sustainable economic development are discussed in [14] with evaluating real options embedded in these projects.

The decision maker makes its decisions based on the changes in factors. These factors vary stochastically according to a certain random process. The idea behind the CRR method is to cover a possible future state variable binomial tree as shown in Figure. 2. This is a role-specific scenario of possible changes in the value of state variables. At each stage, we consider only the possibility of an increase or decrease in value. With this procedure the decision making process is simplified.

This issue is discussed in the literature by Guthrie [9]. Guthrie considered one such factor, and this article extends these considerations and binds the results of the project with two factors.

\section{Method of evaluation}

We assume that each state variable in one period may increase $u$-times and fall $d$-times. This assumption leads to the tree of possible state variable 
values, consisting of nodes marked with indices $(i, j$, $n)$. Each node is denoted as $(i, j, n)$ where

- $\quad i$ - means the number of falls in the first state variable

- $j$ - means the number of falls in the second state variable

- $\quad n$ - is the number of period

A state variable and cash flow is connected to each node. We denote it as (in each node $(i, j, n)$ ) :

- $\quad X_{I}(i, n)$ - first state variable in period $n$

- $X_{2}(j, n)$ - second state variable in period $n$

- $\quad Y^{m}(i, j, n)$ - cash flow in period $n$ (where $m$ is state of project)

With each node is also connected present value of project $V_{s}(i, j, n)$ described later.

We assume that we have $N$ periods, the present value of each state variable denoted by $X_{k}(0,0)$ and also have $u$ and $d$. The value of $u$ can be obtained from historical data by the calibration procedure [9].

The proposed procedure consists of several steps which include: create a decision tree (D-tree), building possible scenarios (X-Tree), build a tree of the project value (V-Tree), and at the end determining effective decisions.

\subsection{Decision tree (D-Tree)}

First of all we must create decision tree (D-Tree). In this step, the possible states of the project are recognized. They may be different phases or specific stages. The possible decisions that could be made when considering a state are recognized. Making a decision would lead to a transition from one state to another. All possible transitions are identified. The result of this step is the creation of a D-Tree. The considered D-Tree is shown in Figure 1.

\subsection{State variables tree (X-Tree)}

Next we must build a lattice of state variables (XTree). In this step, quantifiable economic magnitudes which may depend on the result of the project (state variables) need to be identified. This currently proposed method does not include the correlation between these variables, assuming it does not exist.

The tree starts from a known present value of state variables. Based on the history of changes of this magnitude, the values $u$ and $d$ can be determined in the calibration process. A tree of possible changes in state variables is formed and shows possible scenarios of the situation, presented in Figure. 3. Calibration is an appropriate choice for the number of steps and the choice of parameters $d, u$, so as to best meet the future value of the variable state.
The first step is to choose the model of the stochastic process. In the literature [4], the most commonly used processes are:

- Brownian Motion (BM) - defined by equation:

$d X_{t}=\mu d t+\sigma d W_{t}$

- Geometric Brownian Motion (GBM) defined by equation:

where:

$d X_{t}=\mu X_{t} d t+\sigma X_{t} d W_{t}$

$W_{t}-$ is the Wiener process

$X_{t}-$ is the state variable

$\mu-$ is the drift parameter

$\sigma-$ is the volatility parameter

In literature on the subject [15], are discussed formal methods of model selection. How important is the proper selection of a stochastic process model, shown in Figure 2. This figure shows generated examples of trajectories GBM process, for which was superimposed tree for BM process. It is evident that certain trajectories go beyond the lattice. Model selection implies a way of generating lattice nodes. They are as follows:

- for Brownian Motion (BM) nodes are defined by equation:

$$
X_{1}(i, n)=X_{1}(0,0)+(n-2 i) \cdot \hat{\sigma}_{1} \sqrt{\Delta t_{m}}
$$

- for Geometric Brownian Motion (GBM) nodes are defined by equation:

$$
X_{2}(j, n)=X_{2}(0,0) e^{(n-2 j) \cdot \hat{\sigma}_{2} \sqrt{\Delta t_{m}}}
$$

where:

$\Delta t_{m}$ - is a part of year which represents one period $\hat{\sigma}_{1}-$ is the estimated volatility parameter for BM $\hat{\sigma}_{2}-$ is the estimated volatility parameter for GBM

These formulas arise directly from the method of determining the parameters $u$ and $d$. This is done in the model calibration process. They are as follows:

- for Brownian Motion (BM) we have:

$u=\hat{\sigma}_{1} \sqrt{\Delta t_{m}}$

$d=-\hat{\sigma}_{1} \sqrt{\Delta t_{m}}$

- for Geometric Brownian Motion (GBM) we have:

$$
\begin{aligned}
& u=e^{\hat{\sigma}_{2} \sqrt{\Delta t_{m}}} \\
& d=e^{-\hat{\sigma}_{2} \sqrt{\Delta t_{m}}}
\end{aligned}
$$

The volatility of the process $\hat{\sigma}_{l}$, is in each case calculated on the basis of historical data from the variability in this data: 


$$
\hat{\sigma}_{l}=\frac{\sigma}{\sqrt{\Delta t_{d}}}
$$

where:

$\Delta t_{m}$ - is a part of year which represents one period in data

$\sigma-$ standard deviation in historical data.

\subsection{Tree of the project values (V-Tree)}

Next step is building a tree of the project values. If a project evaluation is based on many state variables, it is therefore presented in a vector of values. When considering two state variables, the VTree grows in two dimensions:

$$
\mathbf{V}^{m}(i, j, n)=\left[\begin{array}{c}
f_{1}\left(X_{1}(i, n), V_{1}^{m}(i, j, n+1)\right) \\
f_{2}\left(X_{2}(j, n), V_{2}^{m}(i, j, n+1)\right)
\end{array}\right]
$$

We denote the present value of the project, which is dependent on two state variables, as:

- $V_{s}(i, j, n)$ - utility value of project in period $n$, with $i$ - number of falls of first state variable and $j$ - number of falls of second state variable

The calculation of the value of the V-Tree starts from the end (final value). We assume that the final value of the project is a function of state variables:

$$
\mathbf{V}^{m}(i, j, N)=\left[\begin{array}{c}
f_{1}\left(X_{1}(i, N)\right. \\
f_{2}\left(X_{2}(j, N)\right.
\end{array}\right]
$$

On this basis, the remaining values of the V-Tree are successively calculated. Trees are constructed for each state of the project. The calculation of value is done by backward induction. Based on the value of the project after its completion (which is usually equal to the state variable, or the right formula for this variable is calculated), the values of the project in the preceding nodes are calculated. Schema of the calculation is shown in Figure. 3.

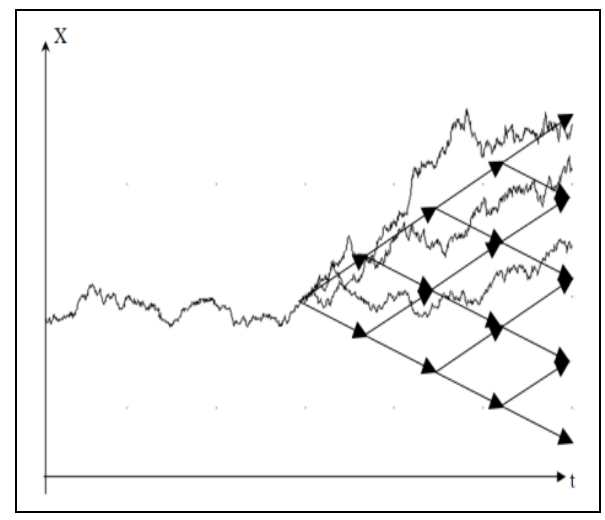

Figure 2. The binomial tree covering stochastic process

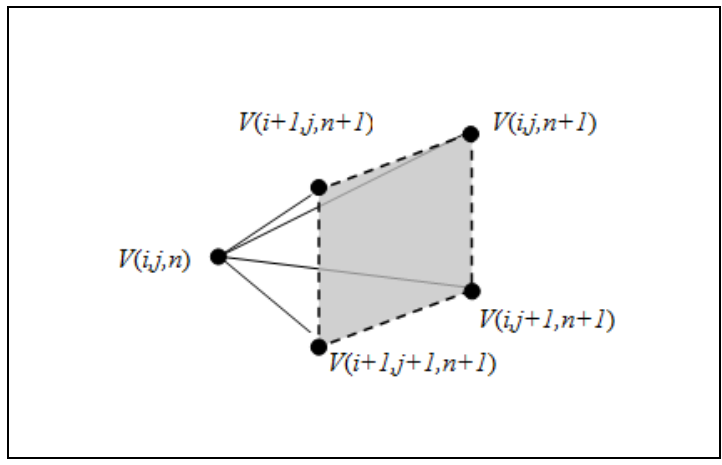

Figure 3. Schema of calculations

We will consider two possibilities:

- in the case of other than financial factors, the present value is the expected value of subsequent values

$$
\begin{aligned}
V_{1}^{m}(i, j, n)= & \pi_{u}^{1} \pi_{u}^{2} V_{1}^{m}(i, j, n+1)+ \\
& +\pi_{u}^{1} \pi_{d}^{2} V_{1}^{m}(i, j+1, n+1)+ \\
& +\pi_{d}^{1} \pi_{u}^{2} V_{1}^{m}(i+1, j, n+1)+ \\
& +\pi_{d}^{1} \pi_{d}^{2} V_{1}^{m}(i+1, j+1, n+1)
\end{aligned}
$$

- in the case of financial factors, when the present value is the discounted expected value of subsequent values

$$
\begin{aligned}
V_{2}^{m}(i, j, n)= & \left(\pi_{u}^{1} \pi_{u}^{2} V_{2}^{m}(i, j, n+1)+\right. \\
& +\pi_{u}^{1} \pi_{d}^{2} V_{2}^{m}(i, j+1, n+1)+ \\
& +\pi_{d}^{1} \pi_{u}^{2} V_{2}^{m}(i+1, j, n+1)+ \\
& \left.+\pi_{d}^{1} \pi_{d}^{2} V_{2}^{m}(i+1, j+1, n+1)\right) e^{-r \Delta t_{m}}
\end{aligned}
$$

Subsequent values are weighted by the probability of achieving those values. If we denote by $r$ the risk free interest rate, we can calculate them from the formulas [16]:

- in case of Brownian Motion (BM) [4] -

$\pi_{u}^{1}=\frac{e^{r \Delta t_{m}} E\left(X_{1}(-, n+1)\right)-X_{1}(i+1, n+1)}{X_{1}(i, n+1)-X_{1}(i+1, n+1)}$

for the growth,

$$
\pi_{d}^{1}=1-\pi_{u}^{1}
$$

for the fall of first state variable

- in case of Geometric Brownian Motion (GBM) we have

$\pi_{u}^{2}=\frac{e^{r \Delta t_{m}}-d}{u-d}$

for the growth and

$\pi_{d}^{2}=\frac{u-e^{r \Delta t_{m}}}{u-d}$

for the fall of second state variable. 


\subsection{Effective decisions}

The last step is determination of effective transitions, which are decisions, in this case. The values determined by the formulas (12) - (13) must be calculated for each decision, so we have a superscript denoting the decision on the value achieved:

$$
\mathbf{V}^{A}(i, j, n)=\left[\begin{array}{l}
V_{1}^{A}(i, j, n) \\
V_{2}^{A}(i, j, n)
\end{array}\right]
$$

for decision Act and

$$
\mathbf{V}^{W}(i, j, n)=\left[\begin{array}{l}
V_{1}^{W}(i, j, n) \\
V_{2}^{W}(i, j, n)
\end{array}\right]
$$

for decision Wait.

Assuming that the project is properly managed, a favorable decision will be chosen.

$$
\text { IF } \begin{aligned}
\mathbf{V}^{W}(i, j, n) \succ & \mathbf{V}^{A}(i, j, n) \\
& \text { THEN Wait } ; \mathbf{V}^{m}=\mathbf{V}^{W} \\
& \text { ELSE Act } ; \mathbf{V}^{m}=\mathbf{V}^{A}
\end{aligned}
$$

In (20) the preference between the two vectors must be checked. This issue was considered in the work [17]. There are many methods for determining the preferred decision based on the preferences of the decision maker. The simplest solution is used in a dynamic programming scalarization approach [18]. The resulting vector components are weighted coefficients indicating preferences. Single numbers are thus obtained, which can be directly compared.

If we denote:

$$
\begin{aligned}
& Q^{W}(i, j, n)=\sum_{k} w_{k} V_{k}^{W}(i, j, n) \\
& Q^{A}(i, j, n)=\sum_{k} w_{k} V_{k}^{A}(i, j, n)
\end{aligned}
$$

where $w_{k} \geq 0$ are weights of assessments, the calculations are simplified to:

$$
\begin{array}{r}
\text { IF } Q^{W}(i, j, n)>Q^{A}(i, j, n) \text { THEN Wait } \\
\text { ELSE Attempt }
\end{array}
$$

After determining that the weights of the criteria are sufficient, the two values are arithmetically compared in order to determine the desired decision.

\section{Numerical example}

We will consider a six-month project of social nature. The project can be started at the beginning of the year. The project can be started at the beginning of the year. It is, however, to delay the start of the half year. This is often the free decision of the project manager involvement in the project. Real option is the decision to make the project and the time of its start. After its implementation financing in the amount of 10 million euros will be obtained.
Costs were estimated at 41 million PLN. In addition, if the project proves to be purposeful further cooperation of the financing institution will be possible. The purposefulness the project depends on the development of the level of unemployment. If it remains high, the project will be deemed purposeful. If the unemployment rate drops, its implementation will be useless.

$\mathrm{X}$-Trees determined on the basis of the observations of variables in 2012 are presented in Table 1 for the level of unemployment and the exchange rate EUR / PLN in Table 2. For the first state variable we use BM, for the second, GBM.

Table 1. X-Tree for unemployment rate

\begin{tabular}{|c|c|r|r|}
\hline$X_{I}$ & \multicolumn{3}{|c|}{$n$} \\
\hline$i$ & 0 & 1 & 2 \\
\hline 0 & 10,4 & 10,8 & 11,2 \\
\hline 1 & & 10,0 & 10,4 \\
\hline 2 & & & 9,6 \\
\hline
\end{tabular}

Table 2. X-Tree for EUR/PLN exchange rate

\begin{tabular}{|c|c|c|c|}
\hline$X_{2}$ & \multicolumn{3}{|c|}{$n$} \\
\hline$J$ & 0 & 1 & 2 \\
\hline 0 & 4,0946 & 4,3487 & 4,6186 \\
\hline 1 & & 3,8553 & 4,0946 \\
\hline 2 & & & 3,6300 \\
\hline
\end{tabular}

The final value of the first state value is calculated as project utility. If unemployment is greater than $10 \%$, this value is 100 , otherwise it is 0 .

$$
f_{1}^{e}\left(X_{1}(i, n)\right)=\left\{\begin{array}{cc}
100 & X_{1}(i, n)>10,0 \% \\
0 & X_{1}(i, n)<=10,0 \%
\end{array}\right.
$$

In the following tables the final values obtained by the project are shown. The final value for the first state value is calculated as project profit :

$$
f_{2}^{e}\left(X_{2}(i, n)\right)=M \cdot X_{2}(j, n)-K
$$

where:

$$
\begin{aligned}
& M=10 \text { M EUR } \\
& K=41 \text { M PLN }
\end{aligned}
$$

For the nodes in Figure. 4 marked with filled circles, we calculate the final results of the project. The calculated final values are presented in Tables 3 to 5 .

Table 3. Final values for $\boldsymbol{n}=\mathbf{2}$ after decision Act

\begin{tabular}{|c|c|c|c|}
\hline$\left(f_{1}^{e} ; f_{2}^{e}\right)$ & \multicolumn{3}{|c|}{$n=2$} \\
& 0 & 1 & 2 \\
\hline$i, j$ & 0 & $(100 ;-0,1)$ & $(100 ;-4,7)$ \\
\hline 0 & $(100 ; 5,2)$ & $(100 ;-0,1)$ & $(100 ;-4,7)$ \\
\hline 1 & $(100 ; 5,2)$ & $(0 ;-0,1)$ & $(0 ;-4,7)$ \\
\hline 2 & $(0 ; 5,2)$ & \multicolumn{3}{|c|}{} \\
\hline
\end{tabular}


Table 4. Final values for $\boldsymbol{n}=\mathbf{2}$ after decision Wait

\begin{tabular}{|c|c|c|c|}
\hline \multirow{2}{*}{$\left(f_{1}^{e} ; f_{2}^{e}\right)$} & \multicolumn{3}{|c|}{$n=2$} \\
& \multicolumn{3}{|c|}{} \\
\hline$i, j$ & 0 & 1 & 2 \\
\hline 0 & $(100 ; 0)$ & $(100 ; 0)$ & $(100 ; 0)$ \\
\hline 1 & $(100 ; 0)$ & $(100 ; 0)$ & $(100 ; 0)$ \\
\hline 2 & $(0 ; 0)$ & $(0 ; 0)$ & $(0 ; 0)$ \\
\hline
\end{tabular}

Table 5. Final values for $n=1$

\begin{tabular}{|c|c|c|}
\hline$\left(f_{1}^{e} ; f_{2}^{e}\right)$ & \multicolumn{2}{|c|}{$n=1$} \\
\hline$i, j$ & 0 & 1 \\
\hline 0 & $(100 ; 2,5)$ & $(100 ;-2,4)$ \\
\hline 1 & $(100 ; 2,5)$ & $(100 ;-2,4)$ \\
\hline
\end{tabular}

Using backward induction, from the equations (12)-(15), we calculate the vectors of values for each decision stage. In the calculations we used the values $r=4 \%, \pi_{u}{ }^{l}=0,5, \pi_{d}{ }^{1}=0,5, \pi_{u}{ }^{2}=0,3725, \pi_{d}{ }^{2}=$ 0,6275 . The results are presented in Tables 7 to 8 .

The dominant elements are marked with an asterisk. If there is no dominant element, the definitive decision can be calculated using the preference structure obtained by weights in the scalarization approach. We assume, that social aspects are more important than financial, so we may take $w_{1}=0,9$ and $w_{2}=0,1$. By comparing the calculated values we get the preferred choice.

Table 6. Decision values for $n=1$

\begin{tabular}{|c|c|c|}
\hline $\begin{array}{c}|c| \\
\left(\mathbf{V}^{A}(i, j, n)\right)^{T} \\
\left(\mathbf{V}^{W}(i, j, n)\right)^{T}\end{array}$ & 0 & 1 \\
\hline$i, j$ & $(100 ; 1,8)^{*}$ & $(100 ;-2,9)$ \\
\hline 0 & $(100 ; 0,0)$ & $(100 ; 0,0)^{*}$ \\
\hline 1 & $(50 ; 1,8)^{*}$ & $(50 ;-2,9)$ \\
& $(50 ; 0,0)$ & $(50 ; 0,0)^{*}$ \\
\hline
\end{tabular}

In all nodes at this period we have dominant decisions, marked with asterisk. And finally for $\mathrm{n}=0$ we have $\left(\mathbf{V}^{A}(i, j, n)\right)^{T}=(75 ; 0,9)$ and $\left(\mathbf{V}^{W}(i, j, n)\right)^{T}=(100 ; 0,0)$ which gives us:

$$
\begin{aligned}
& \mathrm{Q}^{\mathrm{A}}(0,0,0)=0,9 \cdot 75+0,1 \cdot 0,9=67,6 \\
& \mathrm{Q}^{\mathrm{W}}(0,0,0)=0,9 \cdot 100+0,1 \cdot 0,0=90,0
\end{aligned}
$$

It is best to wait with the start of the project by mid-year. Then, if the exchange rate rises, you need to start the project. If the exchange rate falls, do not start the project.

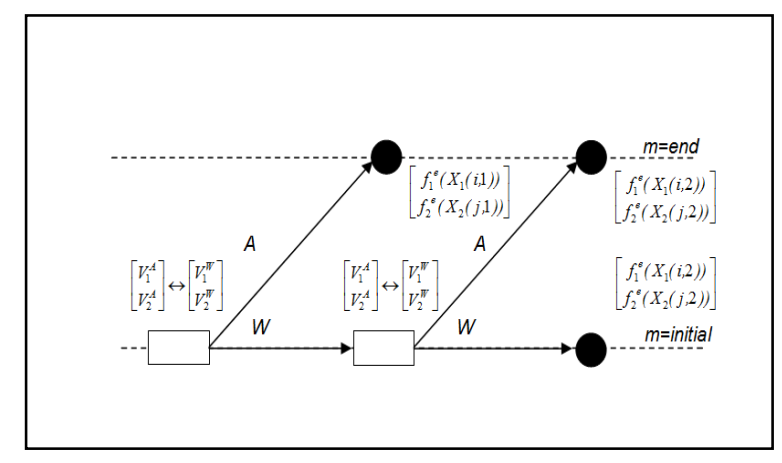

Figure 4. The V-Tree

\section{Conclusions}

The present paper outlines the valuation method of development projects in which real option situations occur. The proposed method allows not only to assess the value of the project but mainly to support decision-making in the project. The method discussed here, as shown in the example, allows not only to make the right decisions about the beginning the project, but also to support decision making during the project's implementation. It does this by determining the appropriate start times of the project.

The method proposed takes into account the dependence of the project on two independent random factors, which are called state variables. Our procedure is based on binomial trees and uses a multicriteria dynamic programming method. The numerical example shows the need of computer implementation of the method. The calculations performed are straightforward but tedious.

The method requires expansion. In the subsequent work of the author intends to include more than two state variables. Further work will be aimed in the direction to take into account the autocorrelation between state variables. It is also necessary computer implementation. A separate topic is the modeling of preferences between state variables. They can be used more advanced methods of multi-criteria decision making.

A novelty of the proposed method is the use of multi-criteria methods in the binomial tree method. Such an approach seems to be particularly significant in projects of sustainable development, which should not only target economic indicators but also take into account social and environmental factors. In a situation where they are difficult to enumerable for the monetary value, multi-criteria approach seems to be most appropriate.

\section{Acknowledgements}


This research was supported by the grant from National Science Centre Poland $(\mathrm{NCN})$, project number NN 111477740.

\section{References}

[1] Black F., Scholes M. (1973) "The Pricing of Options and Corporate Liabilities.", The Journal of Political Economy, vol. 81 (3), pp. 637-654.

[2] Cox, J.C., Ross, S.A., Rubinstein, M. (1979) "Option pricing: a simplified approach", Journal of Financial Economics, vol. 7, pp. 229-263.

[3] Boyle, P.P. (1977) "Options: a Monte Carlo approach", Journal of Financial Economics, vol. 4, pp. 323-338.

[4] Guthrie, G. (2009) Real Options in Theory and Practice. Oxford University Press, Oxford.

[5] Boyle, P.P. (1988) "A Lattice Framework for Option Pricing with Two State Variables", Journal of Financial and Quantitative Analysis, vol. 23 (01), pp. 1-12.

[6] Mun, J. (2010) "Real Options", in J. B. Abrams: Quantitative Business Valuation. Wiley. 2nd ed. Hoboken.

[7] Office of Government Commerce: Managing Successful Projects with PRINCE2 (2009 ed.). TSO, 2009.

[8] Ingersoll J., Ross, S.A. (1992) "Waiting to Invest: Investment and Uncertainty", The Journal of Business, vol. 65 (1), pp. 1-29.

[9] Bednyagin, D., Gnansounou, E. (2012) "Estimating spillover benefits of large R\&D projects: Application of real options modelling approach to the case of thermonuclear fusion R\&D programme", Energy Policy, vol. 41, pp. 269-279.

[10] Comello, S.D., Lepech, M.D., Schwegler B. (2012) "Project-Level Assessment of Environmental Impact: Ecosystem Services Approach to Sustainable Management and Development", Journal Of Management In Engineering, vol 28 (1), pp. 5-12.

[11] Plottu, E., Plottu, B. (2012) "Total landscape values: a multi-dimensional approach", Journal Of Environmental Planning And Management, vol. 55 (6), pp.797-811.

[12] Heidkamp, C.P. (2008) "A theoretical framework for a 'spatially conscious' economic analysis of environmental issues", Geoforum, vol. 39 (1), pp. 62-75.

[13] Evans, R., Moran, C.J., Brereton, D. (2006) "Beyond NPV - A review of valuation methodologies and their applicability to water in mining", in Proceedings of the Water in Mining 06 Conference, Australasian Institute Of Mining And Metallurgy Publication Series, pp. 97-103.

[14] Chang, Ch. Y. (2012) "Critiques of the Existing Techniques for the Evaluation of Renewable Energy Projects", in 2012 IEEE International Technology Management Conference, pp. 151-158.
[15] De Magalhaes Ozorio, L., Shevchenko, P.V., de Lamare Bastian-Pinto, C., (2013). "The Choice of Stochastic Process in Real Option Valuation II: Selecting Multiple Factor Models" Presented at the Real Options. 17th Annual International Conference, London.

[16] Seydel, R.U. (2009) Tools for Computational Finance, Fourth ed, Universitext. Springer, Berlin Heidelberg.

[17] Targiel K.S. (2013) "Multiple criteria decision making in the valuation of real options", Multiple Criteria Decision Making, vol. 8. (in print)

[18] Trzaskalik, T. (1988) Multiobjective Analysis in Dynamic Environment, Publisher of The Karol Adamiecki University of Economics in Katowice, Katowice. 